\title{
Comparison of the efficacy of icotinib in patients with non-small-cell lung cancer according to the type of epidermal growth factor receptor mutation
}

\author{
ZHANG XIAO XUE ${ }^{1}$, WANG XIU WEN ${ }^{1}$, YU ZHUANG $^{2}$, ZANG JIAN HUA $^{3}$ and YANG NI XIA ${ }^{3}$ \\ ${ }^{1}$ Department of Oncology, Qilu Hospital, School of Medicine, Shandong University, Jinan, Shandong 250012; \\ ${ }^{2}$ Department of Oncology, The Affiliated Hospital of Qingdao University Medical College, Qingdao, Shandong 266003; \\ ${ }^{3}$ Department of Oncology, Qingdao Hiser Hospital, Qingdao, Shandong 266033, P.R. China
}

Received May 25, 2015; Accepted November 12, 2015

DOI: $10.3892 / \mathrm{mco} .2016 .956$

\begin{abstract}
Icotinib hydrochloride is a novel epidermal growth factor receptor (EGFR) tyrosine kinase inhibitor (TKI) with preclinical and clinical activity in non-small-cell lung cancer (NSCLC). Exon 19 deletion and L858R point mutation are the most commonly encountered EGFR mutations in NSCLC, and they predict improved clinical outcomes following treatment with icotinib. The objective of this study was to evaluate the differential clinical efficacy of icotinib in patients with exon 19 deletion or L858R point mutation of the EGFR gene. A total of 104 patients with advanced NSCLC, who harbored exon 19 deletion or L858R point mutation of EGFR and were treated with icotinib, were enrolled in this study. The tumor response and progression-free survival were evaluated. There were no significant differences between patients with EGFR exon 19 deletion and those with L858R point mutation who received treatment with icotinib.
\end{abstract}

\section{Introduction}

Non-small-cell lung cancer (NSCLC) is a life-threatening malignancy, exhibiting the highest incidence and mortality rate amongst all types of cancer worldwide (1). Chemotherapy is traditionally the mainstay of treatment for advanced NSCLC, with a median survival of $\sim 10$ months (2), while the emergence of targeted therapy has not only prolonged patient survival, but also significantly improved the patients' quality of life. Gefitinib and erlotinib, the first oral tyrosine kinase inhibitors (TKIs), have led to a significant improvement in the outcome of patients with epidermal growth factor receptor (EGFR) mutations,

Correspondence to: Professor Wang Xiu Wen, Department of Oncology, Qilu Hospital, School of Medicine, Shandong University, 107 Wen Hua West Road, Jinan, Shandong 250012, P.R. China

E-mail: snowstudy@163.com

Key words: non-small-cell lung cancer, icotinib, epidermal growth factor, exon 19 deletion, $L 858 \mathrm{R}$ point mutation with a median survival of 20 months $(3,4)$, as demonstrated in previous clinical studies (5-7). Icotinib, a novel EGFR-TKI, exerted a distinctly inhibitory effect on NSCLC in vivo and in vitro. A phase III clinical study (ICOGEN) demonstrated that icotinib exhibits high efficacy in the re-treatment of advanced NSCLC, compared with gefitinib (8). The efficacy of small-molecule TKIs has been shown to be associated with the EGFR mutation status. Exons 18-21 are the most common mutation sites in EGFR, with exons 19 and 21 being sensitive to targeted drug therapies. According to the subgroup analysis of recent phase III studies, patients with exon 19 deletion who received elotinib exhibited a better response compared with those with exon 21 L858R mutation (9). Furthermore, it was demonstrated by a previous retrospective study that the efficacy of gefitinib in patients harboring exon 19 deletion was comparable to that in patients harboring exon $21 \mathrm{~L} 858 \mathrm{R}$ mutation (10). We retrospectively analyzed the post-treatment survival data of patients who were treated with icotinib, with the aim of further elucidating the association between the two most common EGFR mutations (exon 19 deletion and exon 21 L858R mutation) and the efficacy of icotinib.

\section{Patients and methods}

Patient inclusion criteria. After reviewing the clinical data of stage IIIb and IV NSCLC patients with complete follow-up records, who were admitted to the Affiliated Hospital of Qingdao University Medical College (Qingdao, China) between August, 2012 and August, 2014, 104 patients with EGFR mutations who received icotinib were identified. The inclusion criteria were as follows: (i) Patients with EGFR exon 19 deletion or exon 21 L858R mutation; (ii) at least 1 measurable clinical lesion according to Response Evaluation Criteria in Solid Tumors (RECIST), version 1.1 (11); (iii) patients who did not receive prior treatment with other TKIs; and (iv) an Eastern Cooperative Oncology Group performance status score of $\leq 3$.

Evaluation of efficacy and toxicity of icotinib. Efficacy evaluation was performed 1 month after the administration of icotinib. Patients with response to treatment or stable disease (SD) underwent computed tomography and other 
imaging tests every 2 months, the results of which were used for efficacy evaluation. The treatment response was assessed with RECIST 1.1 as follows: Complete response (CR), partial response (PR), SD and progressive disease. The response rate (RR) included $C R$ and $P R$. The time to response was calculated from the date of initiation of icotinib administration to the date when a clinical response was detected.

The National Cancer Institute Common Toxicity Criteria, version 3 (http://ctep.cancer.gov), were used to evaluate toxicity and grade $0-4$ side effects.

Detection of EGFR mutations. Pyrosequencing was used to assess the EGFR mutation status of patients by collecting 82 biopsy samples and 22 tissue samples. As one of the major EGFR detection methods currently used, pyrosequencing has become the most widely used detection method in clinical practice due to its specificity. The patients' DNA was re-tested by using ADx EGFR Mutations Detection kit (Amoy Diagnostics, Xiamen, China), which recently received State Food and Drug Administration approval for clinical application in mainland China. The kit used the principle of Amplified Refractory Mutation System and covered the 29 EGFR mutation hotspots from exon 18 to 21 . The assay was carried out according to the manufacturer's protocol with the MX3000P quantitative polymerase chain reaction system (Stratagene, La Jolla, CA, USA). The results were determined as positive or negative according to the criteria defined by the manufacturer's instructions. The results of ADx-AMRS were compared with those of direct sequencing. BioAsia (Shanghai, China) examined all the samples used in this study and provided the detection reports.

Follow-up. Information on the progression-free survival (PFS) of the patients was obtained through outpatient visits or telephone follow-up.

Follow-up results were available for all 104 patients. The last follow-up visit was conducted on March 1st, 2015. PFS was defined as the time from the initiation of icotinib administration to treatment failure (death, progressive disease or intolerable toxicity), or the last visit.

Statistical analysis. All statistical analyses were performed using SPSS software for Windows, version 19.0 (SPSS Inc., Chicago, IL, USA). The Fisher's exact test was used to compare baseline characteristics and the RR of patients with different EGFR mutation types. The Kaplan-Meier method was used to draw survival curves and the PFS data between treatment groups were compared using the log-rank test. $\mathrm{P}<0.05$ was considered to indicate statistically significant differences.

\section{Results}

General characteristics of the patients. A total of 104 patients diagnosed with NSCLC harboring EGFR exon 19 deletion or exon 21 L858R mutation between August, 2012 and August, 2014, were enrolled in this study. Of the 104 patients, 49 were male and 55 were female. The median age of all the patients was 62 years. According to the pathological diagnosis, 101 patients were confirmed with adenocarcinoma and 3 had other types of NSCLC. A total of 10 patients were at stage IIIb and the remaining patients at stage IV; among
Table I. Patient characteristics $(\mathrm{n}=104)$.

Characteristics

Patients, no. (\%)

\begin{tabular}{lc}
\hline Gender & \\
Male & $49(47.0)$ \\
Female & $55(53.0)$ \\
Median age, years (range) & $62(42-75)$ \\
ECOG PS & \\
0 or 1 & $67(64.0)$ \\
2 or 3 & $37(36.0)$ \\
Histology & \\
Adenocarcinoma & $101(97.0)$ \\
Not otherwise specified & $3(3.0)$ \\
EGFR mutation & \\
Exon 19 deletion & $60(58.0)$ \\
L858R mutation & $44(42.0)$ \\
Disease stage & $10(9.6)$ \\
IIIB & $84(80.8)$ \\
IV & $10(9.6)$ \\
Recurrence & \\
Number of prior regimens & $65(62.5)$ \\
0 & $39(37.5)$ \\
$\geq 1$ &
\end{tabular}

ECOG PS, Eastern Cooperative Oncology Group performance status; EGFR, epidermal growth factor receptor.

these, 20 patients had recurrent and metastatic NSCLC. Of the 104 patients, 60 harbored an exon 19 deletion and 44 a 21 L858R mutation. The general characteristics of the patients are summarized in Table I. There were significant differences between the NSCLC patients harboring exon 19 deletion and those harboring the L858R point mutation in the distribution of the categorical characteristics, i.e., male predominance (48.3 vs. $45.5 \%, \mathrm{P}=0.843$ ) and poor PS (36.7 vs. $32.5 \%$, $\mathrm{P}=0.831)$.

Clinical efficacy evaluation. Of the 104 patients, an objective response $(\mathrm{PR}+\mathrm{CR})$ was obtained in 64 , including a $\mathrm{CR}$ in 2 patients, representing an RR of $61.5 \%$. The patients achieving $\mathrm{CR}+\mathrm{PR}$ included 38 with exon 19 deletion and 26 with exon 21 L858R mutation, with objective RRs of 63.3 and 59\%, respectively. Table II shows the overall RR of the two groups. There was no significant difference in RR between the two groups $(\mathrm{P}=0.688)$.

Correlation of the EGFR genotype with PFS. The median PFS in the overall patient population was 8.5 months [95\% confidence interval (CI): 7.8-10.4 months]. With regard to the EGFR genotype, the median PFS was 9.1 months (95\% CI: 7.8-11.3 months) in patients with exon 19 deletion and 7.8 months (95\% CI: 6.5-10.4 months) in those with exon 21 L858R mutation. There was no statistically significant difference in PFS between the two genotypes $(\mathrm{P}=0.423$, Fig. 1 , Table III). 
Table II. Response to icotinib according to EGFR genotype.

\begin{tabular}{lccc}
\hline Type of mutation & Responders, $n$ & RR, $\%$ & P-value $^{\text {a }}$ \\
\hline Exon 19 deletion $(n=60)$ & 38 & 63.3 & 0.688 \\
L858R mutation $(n=44)$ & 26 & 59.1 \\
Total $(n=104)$ & 64 & 61.5 \\
\hline
\end{tabular}

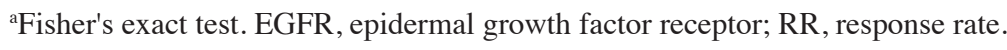

Table III. PFS according to the EGFR genotype.

\begin{tabular}{lccc}
\hline Type of mutation & $\mathrm{n}$ & PFS (months) & $95 \%$ CI \\
\hline Exon 19 deletion & 60 & 9.1 & $7.8-11.3$ \\
L858R mutation & 44 & 7.8 & $6.5-10.4$ \\
\hline
\end{tabular}

PFS, progression-free survival; EGFR, epithelial growth factor receptor; $\mathrm{CI}$, confidence interval.

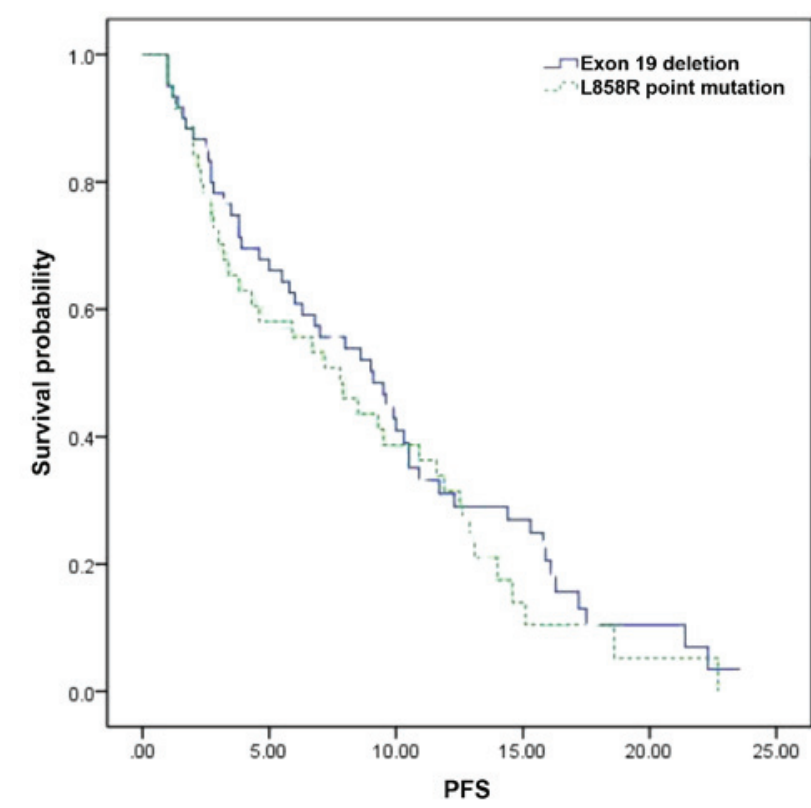

Figure 1. Progression-free survival (PFS) according to the epidermal growth factor receptor genotype.

Toxic and adverse events. The main toxic and adverse events associated with the administration of icotinib were a rash in $41 / 104(39.4 \%)$ and diarrhea in $27 / 104$ patients $(25.9 \%)$. Grade III-IV toxic and adverse events were rarely encountered, with an overall incidence of $10.5 \%(11 / 104)$ in all patients Among these, grade III rash was observed in 6 patients, grade III diarrhea in 4 and grade III fatigue in 1 patient; no patients reported grade IV adverse reactions.

\section{Discussion}

Icotinib is the most commonly used EGFR-TKI in China and the third worldwide after erlotinib and gefitinib. According to the ICOGEN clinical study, icotinib has a similar efficacy to gefitinib $(12,13)$ in the re-treatment of advanced NSCLC and is associated with a significantly lower incidence of adverse events $(8,14)$. Icotinib is currently the standard therapy for advanced NSCLC in China. The median PFS was 10.8 months in this study, indicating that icotinib is effective in the treatment of patients with EGFR mutations. There was no difference in PFS between patients harboring exon 19 deletion and those with exon 21 L858R mutation. The overall survival (OS) data in this study were inconclusive.

Exon 19 deletion and exon 21 L858R mutation are the most common types of EGFR mutation. A European phase III randomized controlled trial of erlotinib (Tarceva $\left.{ }^{\circledR}\right)$ vs. chemotherapy(EURTAC trial)demonstrated that NSCLC patients with exon 19 deletion have a more favorable response rate, PFS and OS compared with patients with exon 21 L858R mutation (9). Based on in vitro cell cultures, another study demonstrated that exon 19 deletion may have a greater affinity for TKIs and display a higher sensitivity to treatment compared with exon 21 L858R mutation (15). However, a retrospective clinical study by Igawa et al (10) revealed no significant differences in RR, PFR or OS between patients with exon 19 deletion and those with exon 21 L858R mutation after receiving gefitinib, which is consistent with the stratification analysis results of another two large, randomized, phase III trials [NEJ002 (12) and WJTOG3405 (13)]. Hence, there remain disputes regarding the correlation between EGFR mutation sites and therapeutic response. Icotinib, a novel targeted agent based on structural modification of erlotinib, differs from gefitinib and erlotinib in its metabolism. In our study, we observed that there were no significant differences in RR and PFS between the two groups and that icotinib imparted a PFS benefit in patients with exon 19 deletion compared with those with exon 21 mutation. Larger samples and a prolonged study duration are required to determine whether there are significant differences in PFS and OS between the two groups treated with icotinib. Further studies are required to determine whether the different responses between the two groups are associated with the mutation or drug configuration.

This study, which conducted a retrospective survival analysis, also has several limitations. First, it was a retrospective study that may contain selection bias. For example, there were minor differences between our study and previous clinical studies in terms of mean age, proportion of females and proportion of adenocarcinoma patients. However, no major differences were found in patient characteristics, e.g., gender and PS score (determined by the Fisher's exact test). Second, gefitinib, erlotinib and icotinib differ in their molecular 
structure. It was previously demonstrated that gefitinib and erlotinib exhibit different efficacy in exon 19 deletion and exon 21 L858R mutation regarding patient outcomes; thus, differences in molecular structures may affect efficacy. Third, there may be no statistical difference in PFS benefit due to the small sample size; the OS results were inconclusive due to the short follow-up duration and the PFS data were censored. These factors may affect the results of this study.

In conclusion, NSCLC patients with exon 19 deletion in the EGFR gene tended to have a better PFS compared with those with exon 21 L858R mutation following treatment with icotinib.

\section{References}

1. Jemal A, Siegel R, Xu J and Ward E: Cancer statistics, 2010. CA Cancer J Clin 60: 277-300, 2010.

2. Schiller JH, Harrington D, Belani CP, Langer C, Sandler A, Krook J, Zhu J and Johnson DH; Eastern Cooperative Oncology Group: Comparison of four chemotherapy regimens for advanced non-small-cell lung cancer. N Engl J Med 346: 92-98, 2002.

3. Shepherd FA, Rodrigues Pereira J, Ciuleanu T, Tan EH, Hirsh V, Thongprasert S, Campos D, Maoleekoonpiroj S, Smylie M, et al: Erlotinib in previously treated non-small-cell lung cancer. N Engl J Med 353: 123-132, 2005.

4. Thatcher N, Chang A, Parikh P, Rodrigues Pereira J, Ciuleanu T, von Pawel J, Thongprasert S, Tan EH, Pemberton K, Archer V and Carroll K: Gefitinib plus best supportive care in previously treated patients with refractory advanced non-small-cell lung cancer: Results from a randomised, placebo-controlled, multicentre study (Iressa Survival Evaluation in Lung Cancer). Lancet 366: 1527-1537, 2005.

5. Lynch TJ, Bell DW, Sordella R, Gurubhagavatula S, Okimoto RA, Brannigan BW, Harris PL, Haserlat SM, Supko JG, Haluska FG, et al: Activating mutations in the epidermal growth factor receptor underlying responsiveness of non-small-cell lung cancer to gefitinib. N Engl J Med 350: 2129-2139, 2004.

6. Paez JG, Jänne PA, Lee JC, Tracy S, Greulich H, Gabriel S, Herman P, Kaye FJ, Lindeman N, Boggon TJ, et al: EGFR mutations in lung cancer: Correlation with clinical response to gefitinib therapy. Science 304: 1497-1500, 2004.

7. Pao W, Miller V, Zakowski M, Doherty J, Politi K, Sarkaria I, Singh B, Heelan R, Rusch V, Fulton L, et al: EGF receptor gene mutations are common in lung cancers from 'never smokers' and are associated with sensitivity of tumors to gefitinib and erlotinib. Proc Natl Acad Sci USA 101: 13306-13311, 2004.

8. Sun Y, Shi Y, Zhang L, et al: A randomized, double-blind phase III study of icotinib versus gefitinib in patients with advanced non-small cell lung cancer (NSCLC) previously treated with chemotherapy (ICOGEN). J Clin Oncol 29 (Suppl) S7522, 2011.
9. Rosell R, Carcereny E, Gervais R, Vergnenegre A, Massuti B, Felip E, Palmero R, Garcia-Gomez R, Pallares C, Sanchez JM, et al: Erlotinib versus standard chemotherapy as first-line treatment for European patients with advanced EGFR mutation-positive non-small-cell lung cancer (EURTAC): A multicentre, open-label, randomised phase 3 trial. Lancet Oncol 13: 239-246, 2012.

10. Igawa S, Kasajima M, Ishihara M, Kimura M, Hiyoshi Y, Asakuma M, Otani S, Katono K, Sasaki J and Masuda N: Comparison of the efficacy of gefitinib in patients with non-small cell lung cancer according to the type of epidermal growth factor receptor. Oncology 87: 215-223, 2014.

11. Eisenhauer EA, Therasse P, Bogaerts J, Schwartz LH, Sargent D, Ford R, Dancey J, Arbuck S, Gwyther S, Mooney M, et al: New response evaluation criteria in solid tumours: Revised RECIST guideline (version 1.1). Eur J Cancer 45: 228-247, 2009.

12. Maemondo M, Inoue A, Kobayashi K, Sugawara S, Oizumi S, Isobe $\mathrm{H}$, Gemma A, Harada M, Yoshizawa H, Kinoshita I, et al: Gefitinib or chemotherapy for non-small-cell lung cancer with mutated EGFR. N Engl J Med 362: 2380-2388, 2010.

13. Mitsudomi T, Morita S, Yatabe Y, Negoro S, Okamoto I, Tsurutani J, Seto T, Satouchi M, Tada H, Hirashima T, et al: Gefitinib versus cisplatin plus docetaxel in patients with non-small-cell lung cancer harbouring mutations of the epidermal growth factor receptor (WJTOG3405): An open label, randomised phase 3 trial. Lancet Oncol 11: 121-128, 2010.

14. Gu A, Shi C, Xiong L, Chu T, Pei J and Han B: Efficacy and safety evaluation of icotinib in patients with advanced non-small cell lung cancer. Chin J Cancer Res 25: 90-94, 2013.

15. Carey KD, Garton AJ, Romero MS, Kahler J, Thomson S, Ross S, Park F, Haley JD, Gibson N and Sliwkowski MX: Kinetic analysis of epidermal growth factor receptor somatic mutant proteins shows increased sensitivity to the epidermal growth factor receptor tyrosine kinase inhibitor, erlotinib. Cancer Res 66: 8163-8171, 2006. 